C. Wihler · S. Schäfer · K. Schmid • E. K. Deemer · G. Münch • M. Bleich •

A. E. Busch - T. Dingermann - V. Somoza - J. W. Baynes - J. Huber

\title{
Renal accumulation and clearance of advanced glycation end-products in type 2 diabetic nephropathy: effect of angiotensin-converting enzyme and vasopeptidase inhibition
}

Received: 19 October 2004 / Accepted: 2 April 2005 / Published online: 12 July 2005

(C) Springer-Verlag 2005

\begin{abstract}
Aims/hypothesis: Renal accumulation of AGEs may contribute to the progression of diabetic nephropathy. We evaluated the effect of ramipril (a pure ACE inhibitor) and AVE7688 (a dual inhibitor of ACE and neutral endopeptidase) on renal accumulation of the advanced glycation end-product (AGE) 3-deoxyglucosone-imidazolone, carboxymethyllysine (CML) and pentosidine, and on clearance of CML in type 2 diabetes. Methods: Male Zucker diabetic fatty rats (ZDF, Gmi-falfa) rats were treated from age 10 to 37 weeks with ramipril $\left(1 \mathrm{mg} \cdot \mathrm{kg}^{-1} \cdot \mathrm{day}^{-1}\right)$, AVE7688 (45 mg $\left.\cdot \mathrm{kg}^{-1} \cdot \mathrm{day}^{-1}\right)$ or without drug. Ramipril and AVE7688 reduced albuminuria by 30 and $90 \%$, respectively. Results: ZDF rats showed increased renal accumulation of the AGE subtypes 3-deoxyglucosone-imidazolone, pentosidine and CML by about 40, 55 and 55\%,
\end{abstract}

C. Wihler · S. Schäfer · M. Bleich · A. E. Busch · J. Huber $(\bowtie)$ Therapeutic Department Cardiovascular, Aventis Pharma Deutschland $\mathrm{GmbH}$, 65926 Frankfurt am Main, Germany e-mail: Jochen.Huber@sanofi-aventis.com Tel.: +49-69-30517124

Fax: +49-69-305942312

K. Schmid · V. Somoza

German Research Institute for Food Chemistry,

Garching, Germany

E. K. Deemer · J. W. Baynes

Department of Chemistry and Biochemistry,

University of South Carolina,

Columbia, SC, USA

G. Münch

Department of Biochemistry and Molecular Biology,

James Cook University,

Townsville, Queensland, Australia

M. Bleich

Christian-Albrechts-University Kiel,

Kiel, Germany

T. Dingermann

Institute for Pharmaceutical Biology,

Johann Wolfgang Goethe University Frankfurt am Main,

Frankfurt am Main, Germany respectively compared with heterozygous, non-diabetic control animals at the age of 37 weeks. AVE7688 but not ramipril attenuated the renal accumulation of 3-deoxyglucosone-imidazolone, pentosidine and CML and improved CML clearance in ZDF rats. During glycation reactions in vitro, AVE7688 also demonstrated potent chelating activity and inhibited metal-catalysed formation of pentosidine and CML. Conclusions/interpretation: Improved AGE clearance and direct inhibition of AGE formation by chelation may contribute to reduced accumulation of renal AGEs and to the nephroprotective effects of vasopeptidase inhibition in type 2 diabetes.

Keywords Advanced glycation end-products ·

Angiotensin-converting enzyme inhibition - AVE7688 .

Diabetes mellitus · Diabetic nephropathy · Ramipril ·

Vasopeptidase inhibition - Zucker diabetic fatty rat

Abbreviations AGE: advanced glycation end-product . CML: carboxymethyllysine - 3-DG: 3-deoxyglucosone ZDF: Zucker diabetic fatty

\section{Introduction}

Diabetic nephropathy is characterised by glomerular, tubular and tubulointerstitial injury, which is mediated by a combination of haemodynamic and metabolic factors [1]. Hyperglycaemia increases oxidative stress, activates the polyol pathway and promotes the formation of various kinds of advanced glyacation end-product (AGE) [2]. AGEs are generated by the non-enzymatic reaction of ketone or aldehyde groups of sugars with free amino groups of proteins, lipids or nucleic acids, the so-called Maillard reaction [3]. AGEs can be formed via oxidative pathways (e.g. carboxymethyllysine [CML] and pentosidine), via non-oxidative pathways (such as pyrraline), or from highly reactive dicarbonyl precursors, such as glyoxal, methylglyoxal and 3-deoxyglucosone (3-DG). Formation of 3-DG-imidazolone is highly specific for the 3DG precursor and thus a marker for the accumulation of 
carbonyl compounds $[4,5]$. The detrimental effects of AGEs in diabetic and non-diabetic nephropathy have been intensively investigated recently [6]. In vivo, CML is the most widely distributed AGE under oxidative conditions and can exert cellular effects by binding to the AGE receptor RAGE [7-9]. Its age-dependent tissue accumulation is accelerated in patients with diabetes mellitus, and serum CML levels correlate with loss of renal function in diabetic patients [10-13]. Some highly reactive AGEs (such as pentosidine) form irreversible cross-links with collagen, which may contribute to structural changes in the kidney [14].

It is well documented that ACE inhibitors are able to delay diabetic nephropathy [15], but the mechanism of action is not clearly understood. Recently, the ACE inhibitor ramipril was demonstrated to reduce renal and serum AGE in a type 1 diabetic, hypertensive rat model [16]. These data provide evidence for a previously unknown mechanism of action of ramipril, beyond its direct inhibition of the formation of angiotensin II. Dual inhibitors of ACE and neutral endopeptidase (or vasopeptidase inhibitors) have shown their potential for even greater nephroprotection compared with ACE inhibition alone in different animal models of nephropathy $[17,18]$.

In the present study, we investigated for the first time the effect of ACE inhibition and vasopeptidase inhibition on the formation, accumulation and excretion of different prototypical AGE subtypes in diabetic nephropathy. We analysed the renal content and excretion of CML, pentosidine and 3-DG-imidazolone after chronic treatment with ramipril (a pure ACE inhibitor) or AVE7688 (a vasopeptidase inhibitor) in Zucker diabetic fatty (ZDF) rats, an animal model of type 2 diabetic nephropathy. In addition to the in vivo studies, we also investigated the effect of ramipril and AVE7688 on AGE formation in vitro and the metal-chelating activity of these drugs.

\section{Materials and methods}

Animal study Male Zucker diabetic fatty rats (ZDF/ Gmi-falfa) and their heterozygous (ZDF/Gmi-+/fa) lean littermates were purchased from Charles River, Sulzfeld, Germany. The animals were housed individually in standard cages in local facilities in Frankfurt-Hoechst. They received standard chow (standard diet 1320; Altromin, Lage, Germany) and tap water ad libitum. All animal experiments were performed in accordance with current Aventis Pharma Laboratory Animal Science and Welfare guidelines and German law for the protection of animals.

ZDF animals were randomly assigned to three groups at the age of 10 weeks. Fifteen ZDF rats did not receive an active pharmacological treatment and were used as a control (ZDF control). A second group $(n=15)$ received $1 \mathrm{mg} \cdot \mathrm{kg}^{-1} \cdot \mathrm{day}^{-1}$ ramipril in drinking water (ZDF ramipril). The third group $(n=14$, ZDF AVE7688) received AVE7688 in standard chow at a concentration of $450 \mathrm{mg} / \mathrm{kg}$. Taking into account an average daily food intake of $40 \mathrm{~g} / \mathrm{rat}$, a dose of approximately $45 \mathrm{mg} \cdot \mathrm{kg}^{-1} \cdot \mathrm{day}^{-1}$ was achieved.
The respective doses were chosen because they resulted in similar inhibition of ACE activity during chronic treatment in ZDF rats [19]. Heterozygous animals without active treatment were used as non-diabetic, lean controls $(n=17)$. Blood was taken for metabolic parameters at different time points from the orbital plexus under light anaesthesia (3.5 vol.\% isoflurane in 34:66 [v/v] $\mathrm{N}_{2} \mathrm{O} / \mathrm{O}_{2}$ ).

At the age of 22 weeks, four animals of each group were killed and the kidneys were frozen in liquid nitrogen for interim AGE analysis. The remaining animals were studied at the age of 37 weeks. First, urine samples were collected over $24 \mathrm{~h}$ in metabolic cages at 37 weeks of age. Thereafter, the rats were anaesthetised with sodium pentobarbital $(50 \mathrm{mg} / \mathrm{kg}$ i.p.). Heart rate and systolic blood pressure were then measured using a catheter-tip micromanometer (Millar Instruments, Houston, TX, USA). After completion of the measurements, arterial blood samples were drawn and the kidneys removed.

Glucose (serum), $\mathrm{HbA}_{1} \mathrm{c}$ and creatinine (serum and urine) were quantified using standard kits (Roche Diagnostics, Mannheim, Germany) on a Hitachi 912E analyser (Hitachi, Mountain View, CA, USA). Urinary albumin was quantified using a fluorescence dye binding assay (Mikro-fluoral; Progen Biotechnik, Heidelberg, Germany).

Tissue AGE analysis by dot-blot Kidney samples were immediately frozen in liquid nitrogen and ground for 5 min in liquid nitrogen using a Freezer mill 6750 (C3 Analysetechnik, Haar, Germany). Ten milligrams of the frozen organ powder was dissolved in $1 \mathrm{ml}$ PBS buffer, $\mathrm{pH} 7.4$, containing $0.5 \mathrm{~g} / 1$ Tween-20, $0.5 \mathrm{mmol} / \mathrm{l}$ phenylmethylsulphonyl fluoride (Sigma-Aldrich Chemie, Steinheim, Germany) and $1 \mu \mathrm{g} / \mu \mathrm{l}$ aprotinin (Roche Diagnostics). Samples were vortexed and treated with ultrasound (Sonoplus HD 2070; Bandolin, Berlin, Germany) twice for $5 \mathrm{~s}$ to disintegrate cells. Cell debris was removed by centrifugation.

The protein concentration of the resulting supernatant was determined with a BSA standard using the Lowry method (DC protein assay; Bio-Rad Laboratories, Hercules, CA, USA).

For dot-blot analysis, $10 \mu \mathrm{g}$ kidney protein of each sample (duplicates) was transferred under vacuum to a nitrocellulose membrane (Schleicher \& Schuell, Dassel, Germany). AGE were detected with monoclonal AGEspecific antibodies (anti-CML 011, anti-3-DG-imidazolone JNH-27, anti-pentosidine 012; Biologo, Kronshagen, Germany) at a concentration of $0.25 \mu \mathrm{g} / \mathrm{ml}$. Conjugates were visualised with anti-mouse alkaline phosphatase antibody (1:20,000; Dianova, Hamburg, Germany) and enhanced chemofluorescence detection (Amersham Pharmacia Biotech, High Wycombe, UK) using a Fluorimager 595 (Amersham Pharmacia Biotech). For data analysis, Image-Quant software (Molecular Dynamics, Amersham, Buckinghamshire, England) was used.

CML analysis by HPLC fluorescence Because AGE analysis by dot-blot was only possible for kidney samples, we used HPLC fluorescence to detect CML in serum 
and urine samples from the 37 -week-old animals. In addition, kidney samples from 22- and 37-week-old animals were also analysed by HPLC fluorescence to compare dotblot and HPLC results. For sample hydrolysis, $500 \mu \mathrm{l}$ of urine or serum samples were incubated with $500 \mu \mathrm{l}$ sodium borate buffer $(0.04 \mathrm{~mol} / \mathrm{l}, \mathrm{pH} 9.5)$ and $500 \mu \mathrm{l}$ sodium borohydride $(1 \mathrm{~mol} / 1$ in $0.1 \mathrm{~mol} / 1 \mathrm{NaOH})$, whereas kidney samples were reduced by the use of $100 \mathrm{mg}$ organ powder and incubation with $1 \mathrm{ml} 0.04 \mathrm{~mol} / \mathrm{l}$ sodium borate buffer and $1 \mathrm{ml} 1 \mathrm{~mol} / \mathrm{l}$ sodium borohydride. The samples were incubated for $4 \mathrm{~h}$ at room temperature. Afterwards, $10 \mathrm{ml}$ hydrochloric acid (concentrated $\mathrm{HCl}$; Fisher Scientific, Loughborough, UK) was added to the urine and kidney samples or $1.5 \mathrm{ml}$ concentrated $\mathrm{HCl}$ was added to the serum samples. Hydrolysis was performed in screwcapped flasks at $110^{\circ} \mathrm{C}$ for $20 \mathrm{~h}$, sealed under nitrogen. After hydrolysis, hydrochloric acid was removed by rotary evaporation and samples were resolved in sodium borate buffer $(0.2 \mathrm{~mol} / \mathrm{l}, \mathrm{pH} 9.5)$ followed by membrane filtration (diameter $0.45 \mu \mathrm{m}$; Millex-HV, Millipore, Schwalbach, Germany).

Sample derivatisation for HPLC fluorescence measurement was done as described previously [20].

Renal CML clearance was calculated from serum and urinary $\mathrm{CML}$ as:

CML clearance $=C_{\mathrm{CML} \text { urine }} \times \stackrel{\circ}{V} / C_{\mathrm{CML} \text { serum }} / m$

where $C_{\mathrm{CML}}$ is the concentration of CML in urine and serum $(\mu \mathrm{g} / \mathrm{ml})$, respectively, $V$ is the urinary flow rate $(\mathrm{ml} /$ h) and $m$ is body weight $(\mathrm{kg})$.

Model in vitro studies on AGE inhibition BSA-AGEs were produced by incubation of BSA $(10 \mathrm{mg} / \mathrm{ml}$; SigmaAldrich) with $30 \mathrm{mmol} / 1$ ribose in $200 \mathrm{mmol} / 1$ sodium phosphate buffer, $\mathrm{pH} 7.4$, at $37^{\circ} \mathrm{C}$ for 5 days. To study the effect of ramipril and AVE7688 on BSA-AGE formation, their respective active metabolites ramiprilat and AVE8048 were added to the mix at $1.25,2.5,5$ or $10 \mathrm{mmol} /$ 1. As negative control, BSA alone was also incubated at $37^{\circ} \mathrm{C}$ for 5 days in $200 \mathrm{mmol} / 1$ sodium phosphate buffer. BSA and BSA-AGE from each sample were analysed using the Western blotting method. Samples were resolved by SDS-PAGE and transferred onto a nitrocellulose membrane (Amersham). AGE modification was detected by monoclonal primary antibodies specific for AGEs (antiCML 011, anti-3-DG-imidazolone JNH-27, anti-pentosidine 012; Biologo, Kronshagen, Germany; $0.25 \mu \mathrm{g} / \mathrm{ml}$ ). Conjugates were visualised using anti-mouse horseradishperoxidase-linked antibody (Cell Signaling, Beverly, MA, USA) and subsequent enhanced chemiluminescence detection (Pierce, Rockford, IL, USA) using a FluorS Imager (Bio-Rad). Computer-assisted densitometric analysis was done using Image Quant software (Bio-Rad).

To normalise the Western blotting results, the same amount of protein was loaded onto each lane, and the amount of protein loaded in each lane was determined by Ponceau S staining (Sigma-Aldrich). Ribose-modified
BSA was quantified by computer-assisted densitometric analysis using the Image Quant software (Bio-Rad).

Evaluation of chelating activity of ACE and vasopeptidase inhibitors We assessed the chelating activity of various compounds by determining the concentration required for half-maximal inhibition of the rate of copper-catalysed auto-oxidation of ascorbate. Briefly, the compounds were dissolved in metal-free (Chelex-treated) phosphate buffer $(50 \mathrm{mmol} / \mathrm{l}, \mathrm{pH} 7.4)$ containing $1.5 \mu \mathrm{mol} / 1 \mathrm{CuCl}_{2}$ and $500 \mu \mathrm{mol} / 1$ ascorbate (final concentrations), as described previously [21]. These conditions yielded a halflife of $\sim 60 \mathrm{~min}$ for ascorbate. Aliquots were taken at 0 , 30, and $60 \mathrm{~min}$ and added to HPLC vials containing $1 \mathrm{mmol} / 1$ diethylenetriaminepentaacetic acid. Ascorbate concentration was then measured by reverse phase-HPLC with absorbance detection $(244 \mathrm{~nm})$ and plotted as log percentage ascorbate remaining in order to estimate the half-life of ascorbate. Test compounds were prepared at $30 \mathrm{mmol} / 1$ concentration in DMSO (stock solution) and diluted in $50 \mathrm{mmol} / \mathrm{l}$ phosphate buffer to yield the final concentration. DMSO was adjusted to $1.7 \%(\mathrm{v} / \mathrm{v})$ in all reactions to correct for minor effects on DMSO on the rate of oxidation of ascorbate. The analysis of the data is summarised in the first Table.

Materials Ramipril and AVE7688 were manufactured by Aventis Pharma. The chemical name of AVE7688 is 7-[[(2S)-2-(acetylthio)-1-oxo-3-methylpropyl]amino]-1,2,3, 4,6,7,8,12b-octahydro-6-oxo-(4S,7S,12bR)-pyrido[2,1-a][2] benzapin-4-carboxy-acid, and its molecular weight is $432.5 \mathrm{~g} / \mathrm{mol}$ [22]. AVE8048 is the active thiol form of AVE7688. It is the major metabolite of AVE7688 in vivo and is similarly active against ACE and neutral endopeptidase in vitro. AVE8048 was also synthesised by Aventis Pharma. Ramiprilat is the active diacid metabolite from ramipril formed by cleavage of the ester group.

All other reagents were purchased from commercial suppliers in analytical grade quality.

Statistics Statistical comparisons between the different groups were carried out using unpaired two-sided $t$-tests. The lean control and the active treatment groups were tested against the ZDF control. In vitro AGE inhibition of AVE7688 and ramiprilat was tested against the control, which was not treated with any compound. A $p$ value of less than 0.05 (after correction for multiple comparisons) was considered significant.

\section{Results}

Functional parameters In the homozygous ZDF rats, diabetes mellitus was established at age 27 weeks $\left(\mathrm{HbA}_{1} \mathrm{c}>\right.$ 8.5). At age 37 weeks the serum glucose and $\mathrm{HbA}_{1} \mathrm{c}$ levels were increased in ZDF rats $(p<0.01)$. Serum glucose was unchanged in the AVE7688 group and slightly decreased in the ramipril group at 37 weeks of age, compared with the ZDF controls (Table 1). Development of 
Table 1 Metabolic and renal functional parameters at the age of 37 weeks

Data are mean \pm SEM ${ }_{p}<0.01$ vs ZDF control, ${ }^{\mathrm{b}} p<0.05$ vs $Z \mathrm{DF}$ control $n=10-13$ per group
Lean control ZDF control ZDF AVE7688 ZDF ramipril

\begin{tabular}{lcccc}
\hline Serum glucose $(\mathrm{mmol} / \mathrm{l})$ & $15.48 \pm 1.0^{\mathrm{a}}$ & $44.87 \pm 1.26$ & $45.32 \pm 2.01$ & $40.88 \pm 1.40^{\mathrm{b}}$ \\
$\mathrm{HbA}_{1} \mathrm{c}(\%)$ & $4.7 \pm 0.03^{\mathrm{a}}$ & $10.1 \pm 0.3$ & $12.0 \pm 0.6^{\mathrm{b}}$ & $9.9 \pm 0.4$ \\
Urinary flow $\left(\mathrm{ml} \cdot \mathrm{kg}^{-1} \cdot \mathrm{h}^{-1}\right)$ & $0.8 \pm 0.04$ & $15.1 \pm 1.4$ & $19.2 \pm 1.6$ & $16.2 \pm 1.3$ \\
Urinary albumin excretion $\left(\mathrm{mg} \cdot \mathrm{kg}^{-1} \cdot \mathrm{h}^{-1}\right)$ & $0.08 \pm 0.01^{\mathrm{a}}$ & $14.23 \pm 2.32$ & $1.49 \pm 0.55^{\mathrm{a}}$ & $10.11 \pm 2.17$ \\
Creatinine clearance $\left(\mathrm{ml} \cdot \mathrm{kg}^{-1} \cdot \mathrm{h}^{-1}\right)$ & $268 \pm 13$ & $271 \pm 16$ & $269 \pm 14$ & $328 \pm 19^{\mathrm{b}}$ \\
\hline
\end{tabular}

diabetes was accompanied by a progressive increase in albuminuria in ZDF controls (Table 1). Treatment with ramipril reduced this diabetes-induced increase in albumin excretion by approximately 30\%. Urinary albumin excretion of ZDF rats at the age of 37 weeks was $14.23 \pm$ $2.32 \mathrm{mg} \cdot \mathrm{kg}^{-1} \cdot \mathrm{h}^{-1}$, compared with $10.11 \pm 2.17 \mathrm{mg} \cdot \mathrm{kg}^{-1} \cdot \mathrm{h}^{-1}$ in the ramipril-treated group. AVE7688 completely prevented the development of albuminuria in ZDF rats $(1.49 \pm$ $0.55 \mathrm{mg} \cdot \mathrm{kg}^{-1} \cdot \mathrm{h}^{-1}$ in the AVE7688-treated group, $p<0.01$ vs ZDF controls; Table 1).

The ZDF control rats had lower heart rates than their lean littermates ( $232 \pm 14$ vs $294 \pm 11$ bpm, $p<0.05)$, but systolic pressure was not different $(111 \pm 6$ vs $103 \pm 8 \mathrm{mmHg}$, $p>0.05)$. Heart rate and blood pressure in both ZDF groups with active treatment were not different from those in the ZDF control group.

$C M L$, pentosidine and 3-DG-imidazolone content in the kidney Accumulation of the three AGE subtypes CML, pentosidine and 3-DG-imidazolone was observed in the kidney of ZDF rats. In the dot-blot analysis, signal intensity increased from week 22 to 37 in ZDF rats by $23 \%$ for CML $(p<0.05), 40 \%$ for pentosidine $(p<0.05)$ and $17 \%$ for 3-DG-imidazolone. AVE7688 treatment reduced these AGEs at 37 weeks by another 47,28 or $45 \%$ below the levels of 22-week-old rats. At 22 weeks of age, ZDF rat kidneys had $15-30 \%$ higher and at 37 weeks of age $30-$ $50 \%$ (significantly) higher concentrations $(p<0.01)$ of 3 DG-imidazolone, pentosidine and CML compared with lean control rats (Fig. 1). Ramipril treatment did not reduce 3-DG-imidazolone or pentosidine in 22- or 37-weekold ZDF rats, respectively (Fig. 1), but it significantly reduced renal CML in 37-week-old ZDF rats by $20 \%$ compared with ZDF control rats $(p<0.01)$. AVE7688 reduced 3-DG-imidazolone, pentosidine and CML content in the kidney of 22-week-old ZDF rats by about $20-30 \%$ $(p<0.05$ vs ZDF control). In 37-week-old AVE7688treated ZDF rats, 3-DG-imidazolone, pentosidine and CML were reduced by about $60 \%$, which was below the level in lean control rats $(p<0.01$ vs ZDF control). Thus, long-term treatment with AVE7688 was more effective in reducing 3-DG-imidazolone, pentosidine and CML than short-term intervention.

CML detection with dot-blot was confirmed by HPLC fluorescence using a CML standard (data not shown). Both data sets showed comparable results, with a correlation coefficient of $r=0.8$ and $p<0.001$.

CML content in serum and urine Serum CML was increased approximately twofold in ZDF rats compared with lean controls $(0.26 \pm 0.05$ vs $0.42 \pm 0.10 \mu \mathrm{g} / \mathrm{ml}, p<0.05)$. Treatment with ramipril indicated a trend towards reduction of serum CML $(0.33 \pm 0.06 \mu \mathrm{g} / \mathrm{ml})$, but this change was not statistically significant (Fig. 2a). In comparison, AVE7688 completely inhibited this diabetes-induced increase in serum CML $(0.22 \pm 0.06 \mu \mathrm{g} / \mathrm{ml}, p<0.01$ vs ZDF control; Fig. 2a).

Increased serum CML levels in $\mathrm{ZDF}$ rats were associated with increased concentrations of CML in the urine. HPLC fluorescence measurement of CML in urine indicated high CML excretion in 37-week-old ZDF rats $(1.30 \pm 0.26 \mu \mathrm{g} / \mathrm{ml}, p<0.01)$. Ramipril significantly reduced urinary CML excretion to $0.92 \pm 0.28 \mu \mathrm{g} / \mathrm{ml}(p<0.05$ vs ZDF control) in 37-week-old rats and AVE7688 showed a similar effect $(0.84 \pm 0.13 \mu \mathrm{g} / \mathrm{ml}, p<0.01$ vs ZDF control; Fig. 2b).
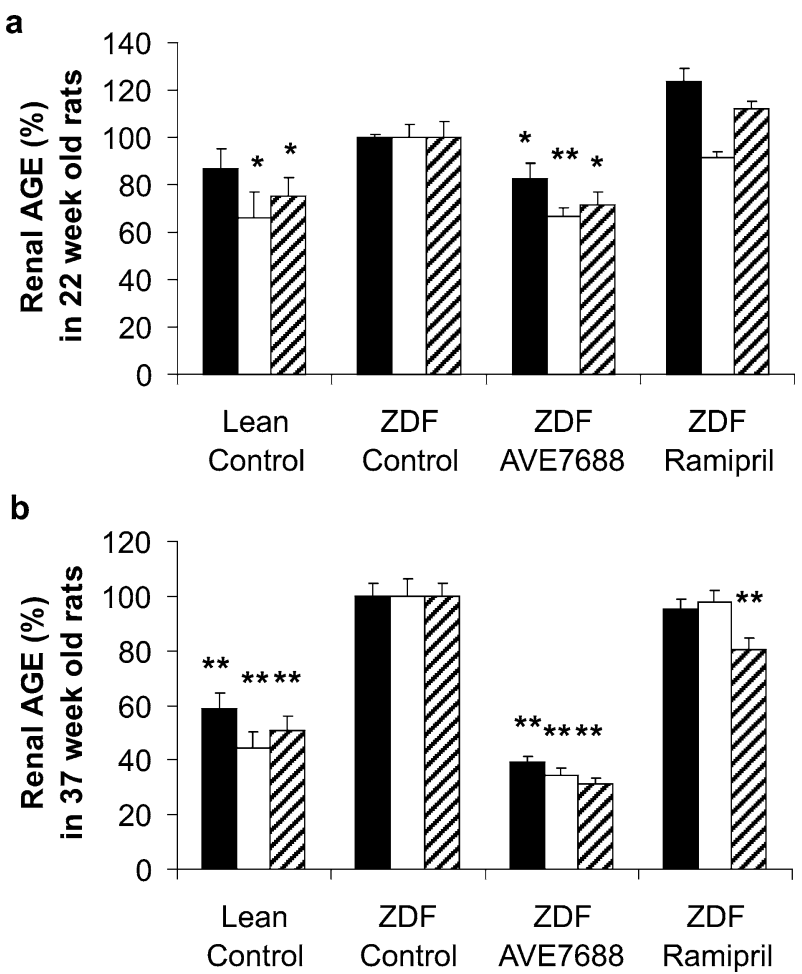

Fig. 1 Dot-blot analysis of AGE accumulation in the kidney. The AGE 3-DG-imidazolone (black bars), pentosidine (white bars) and CML (hatched bars) accumulated in the kidney of ZDF rats aged 22 weeks (a) and 37 weeks (b). This accumulation was prevented at both time points by treatment with AVE7688, while ramipril had no effect on 3-DG-imidazolone or pentosidine but led to a reduction of CML in 37-week-old rats $(\mathbf{a}, \mathbf{b})$. Data are presented as percentage of ZDF controls. ${ }^{*} p<0.05$ vs ZDF control, $* * p<0.01$ vs ZDF control ( $n=4$ for 22-week-old animals, $n=10-13$ for 37 -week-old animals) 
a

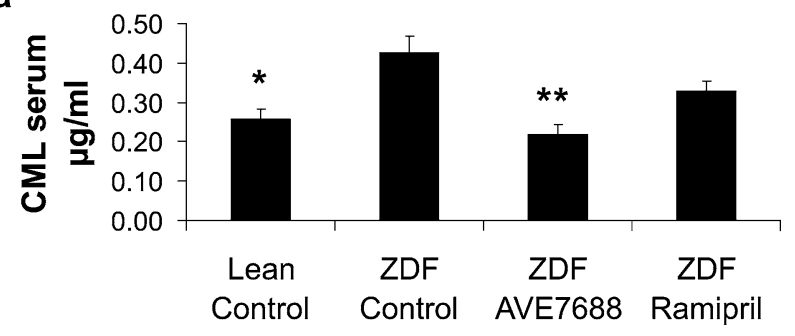

b

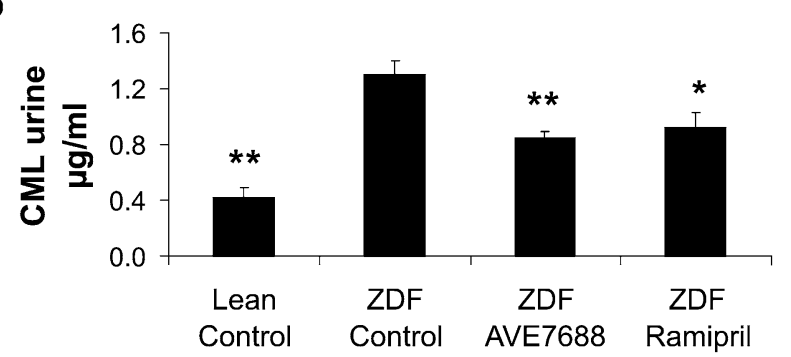

C

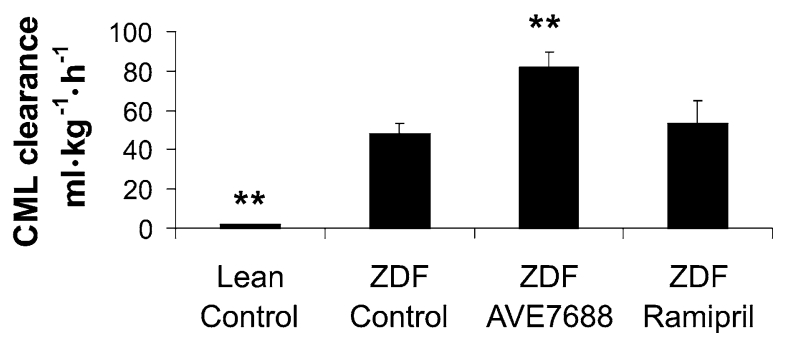

Fig. 2 CML in serum and urine. AVE7688-treated 37-week-old ZDF rats showed decreased CML concentrations in serum (a) and urine (b) as well as improved CML clearance (c). Total CML concentrations were determined by HPLC fluorescence. ${ }^{*} p<0.05$ vs ZDF control, $* * p<0.01$ vs ZDF control ( $n=4-8$ animals $)$

In the ramipril group, CML clearance was not changed while creatinine clearance was slightly increased (Fig. 2c, Table 1). In contrast, AVE7688 increased the serum CML clearance from $48.18 \pm 10.97 \mathrm{ml} \cdot \mathrm{kg}^{-1} \cdot \mathrm{h}^{-1}$ in the control group to $82.06 \pm 16.89 \mathrm{ml} \cdot \mathrm{kg}^{-1} \cdot \mathrm{h}^{-1}(p<0.01$ vs ZDF control) in 37-week-old ZDF rats (Fig. 2c). This change was observed in the absence of a difference in creatinine clearance (Table 1).

In vitro AGE inhibition studies with ramipril and AVE7688 For all in vitro studies, we used the active metabolite of ramipril or AVE7688 (ramiprilat, AVE8048). AGE formation was induced by incubation of BSA with ribose at $37^{\circ} \mathrm{C}$ for 5 days. Pentosidine and $\mathrm{CML}$ formation was detected by western blot analysis using specific monoclonal antibodies. Unmodified BSA incubated at $37^{\circ} \mathrm{C}$ for 5 days was free of any pentosidine or CML modification (Fig. 3a). No 3-DG-imidazolone could be detected in ribose-BSA preparations using a monoclonal 3-DGimidazolone antibody (data not shown).

Ramiprilat had no effect on CML or pentosidine formation, while incubation with AVE8048 (1.25-10 mmol/l) inhibited formation of CML and pentosidine in a concentration-dependent manner (Fig. 3a-c). Pyridoxamine also a

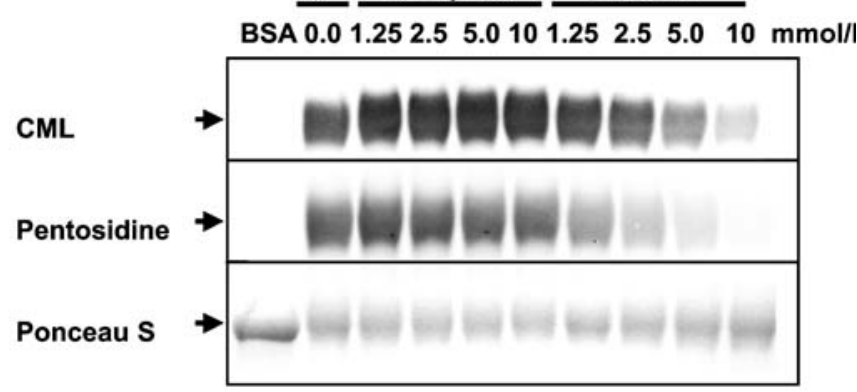

b

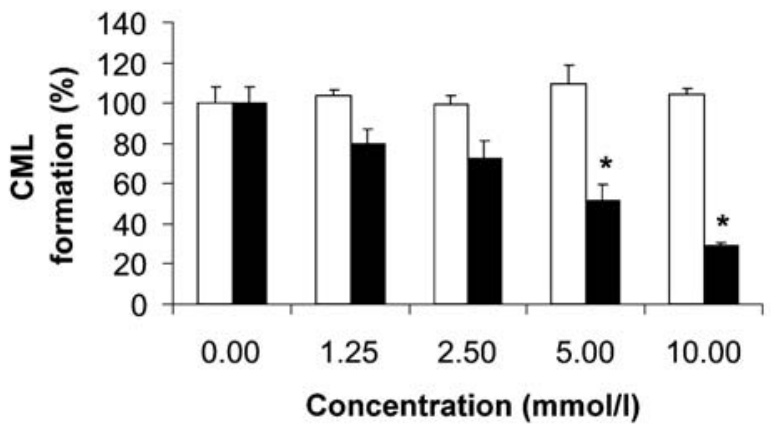

c

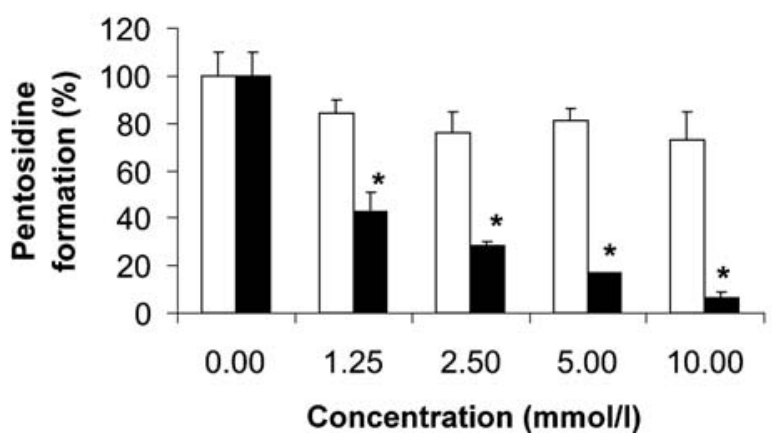

Fig. 3 Effects of AVE8048 and ramiprilat on in vitro AGE formation. Formation of CML and pentosidine was determined by western blotting (a) and is reported as relative percentage of the appropriate control (ribose/BSA without compound) normalised to the amount of loaded protein visualised by Ponceau $S(\mathbf{b}, \mathbf{c})$. AVE8048 (black bars), the active metabolite of AVE7688, inhibits in vitro CML $(\mathbf{a}, \mathbf{b})$ and pentosidine $(\mathbf{a}, \mathbf{c})$ formation from BSA with ribose, while ramiprilat (white bars) shows no effect $(\mathbf{a}, \mathbf{b}, \mathbf{c})$. No CML or pentosidine modification was found in unmodified BSA (a). ${ }^{*} p<0.05$ vs control without drug; $n=3$ for all experiments

showed dose-dependent inhibition of CML formation (data not shown).

Inhibition of metal-catalysed oxidation of ascorbic acid As summarised in Table 2, all of the compounds tested demonstrated chelating activity, based on inhibition of metal-catalysed oxidation of ascorbate. Pyridoxamine and ramiprilat were relatively weak inhibitors, requiring concentrations of $1 \mathrm{mmol} / 1$ and $3-5 \mathrm{mmol} / 1$ for $50 \%$ inhibition of the rate of ascorbate oxidation, respectively. In contrast, the estimated $\mathrm{IC}_{50}$ for AVE8048 was $<10 \mu \mathrm{mol} / 1$, indicating strong metal-chelating activity (Table 2). Thus, the copper binding constant for AVE8048 at physiological $\mathrm{pH}$ was approximately 500 -fold lower (stronger) than that 
Table 2 Chelating activity of pyridoxamine, ramiprilat and AVE8048

\begin{tabular}{ll}
\hline $\begin{array}{l}\text { Drug concentration } \\
(\mathrm{mmol} / \mathrm{l})\end{array}$ & \begin{tabular}{l} 
Ascorbate remaining at $60 \mathrm{~min}(\%)$ \\
\cline { 2 - 2 } Control $^{\mathrm{a}}$ Pyridoxamine Ramiprilat AVE8048
\end{tabular}
\end{tabular}

38

\begin{tabular}{llll}
0.01 & & & $96 \pm 1.33$ \\
0.1 & 42 & & $97 \pm 1.33$ \\
0.5 & 45 & & $99 \pm 0.64$ \\
1.0 & 50 & $38 \pm 0.23$ & $99 \pm 0.46$ \\
5.0 & 66 & $59 \pm 0.40$ & $98 \pm 2.02$ \\
\hline
\end{tabular}

The chelating activity was monitored using the ascorbate assay (see Materials and methods)

Data for various compounds are shown as mean \pm SEM of three independent incubations; data for pyridoxamine are based on longterm quality control measurements and vary by less then $10 \%$ of reported values

${ }^{a}$ The control incubation contained only copper and ascorbate in phosphate buffer; the calculated first-order rate constant $\left(k_{1}\right)$ for oxidation of ascorbate was $0.016 / \mathrm{min}$. The concentration required for $50 \%$ inhibition of copper-catalysed oxidation of ascorbate $\left(\mathrm{IC}_{50}\right)$ was estimated graphically as the amount of the compound required to inhibit the first-order rate of oxidation of ascorbate by $50 \%$, yielding $k_{1}=0.008 / \mathrm{min}$ or $61 \%$ of ascorbate remaining at $60 \mathrm{~min}$. These calculations yielded an $\mathrm{IC}_{50}$ of approximately $3 \mathrm{mmol} / \mathrm{l}$ for pyridoxamine, $3-5 \mathrm{mmol} / \mathrm{l}$ for ramiprilat and $<10 \mu \mathrm{mol} / 1$ for AVE8048

of ramiprilat. None of these compounds had any effect on the oxidation of ascorbate in the absence of copper, nor did they alter the pseudo-first-order kinetics of the autooxidation reaction (data not shown).

\section{Discussion}

Increased production and tissue accumulation of AGEs have been linked to diabetic end organ damage including diabetic nephropathy [23]. Consequently, any intervention which decreases or prevents the deposition of AGEs in the diabetic kidney offers the potential to attenuate the progressive loss of kidney function in diabetic patients.

We have previously shown that chronic vasopeptidase inhibition by AVE7688 prevents diabetic nephropathy in ZDF rats $[19,22]$. The present study demonstrates that AVE7688 also prevents accumulation of the AGEs CML, pentosidine and 3-DG-imidazolone in the diabetic kidney, which offers a mechanistic explanation for the profound nephroprotective effects of vasopeptidase inhibition in the diabetic kidney. Moreover, as all three representative AGEs were reduced by AVE7688, the protective effect of vasopeptidase inhibition was broad-based and not limited to a single pathway, such as oxidative, non-oxidative, or polyoldependent formation of AGEs.

Since AGEs are a heterogeneous group of compounds and contribute to the development of diabetic nephropathy, the three well-characterised AGEs CML, pentosidine and 3-DG-imidazolone were investigated. ZDF rats showed a hyperglycaemia-induced increase in renal CML, pentosidine and 3-DG-imidazolone, indicating broad-spectrum effects on the chemistry and detoxification of reactive carbonyl compounds in diabetes. Elevated renal accumulation of CML, pentosidine and 3-DG-imidazolone in diabetes and diabetic nephropathy has also been reported in other studies [24, 25]. However, this is the first time that an increase in all three AGE subtypes has been demonstrated in ZDF rats, an increase that was suppressed simultaneously by drug intervention. In patients with diabetic nephropathy, urinary excretion of CML is reduced, and insufficient clearance may result in increased serum AGE concentrations as well as tissue accumulation of AGEs [26-29]. This is further supported by the fact that the AGE inhibitor aminoguanidine improved renal function in diabetic nephropathy, reduced tissue AGE deposits [30-32] and increased urinary excretion of dietary AGEs [33]. In the present study, we observed increased CML clearance in the AVE7688 group, indicating that enhanced excretion may contribute to the reduction of plasma and tissue levels of CML. Moreover, this effect may be of clinical relevance, particularly when the endogenous and/or exogenous supply of AGEs is increased and glomerular filtration is impaired, such as in advanced stages of diabetic nephropathy $[34,35]$. The clearance of CML may be influenced by changes in renal filtration, secretion, and reabsorption [36]. Interestingly, CML clearance was increased without changes in glomerular filtration rate, indicating that specific regulation of renal handling of CML may be involved. It may be argued that the reduced AGE accumulation is the consequence rather than the cause of nephroprotection. In fact, nephroprotective agents often reduce proteinuria and AGE accumulation at the same time. However, the specific pathogenetic role of AGEs has been established in animals fed a high vs low AGE diet [37], and pharmacologically using a specific AGE crosslink breaker or AGE inhibitors $[32,38,39]$. Therefore, although we cannot prove directly the causal link between reduced AGE content and proteinuria in the present study, it seems that the reduction of tissue AGEs, e.g. via increased excretion, contributes to the AVE7688-mediated nephroprotection. Moreover, because pure ACE inhibition by ramipril did not influence the CML clearance, it is tempting to speculate that mechanisms related to the inhibition of neutral endopeptidase are responsible for this effect.

The ACE inhibitor ramipril has been demonstrated previously to exert strong nephroprotective effects in diabetic patients [15]. A recent study in a type 1 diabetic animal model [16] also revealed that reduction of albuminuria by ACE inhibition was associated with decreased AGE fluorescence. Although specific AGE were not analysed in this study, the protective effect was similar to that of the AGE inhibitor aminoguanidine [16]. Although we used similar doses of ramipril in our study, ramipril only slightly reduced renal CML accumulation and decreased albuminuria by $30 \%$. Differences in the disease models (type 1 vs type 2 diabetes), resulting in different body composition and, hence, drug metabolism and pharmacokinetics, as well as differences in methods for AGE measurement, may explain the quantitative differences between our work and that of Forbes and colleagues [16]. Inter- 
estingly, the nephroprotective effect of ramipril in type 2 diabetic nephropathy seems to be limited to the reduction of CML accumulation; pentosidine and 3-DG-imidazole were not affected. Together with the modest (approximately $30 \%$ ) reduction in albuminuria by ramipril, it is tempting to speculate that the superior nephroprotection mediated by vasopeptidase inhibition is related to the broader effects of AVE7688 on AGE clearance and accumulation. The current dose of ramipril $\left(1 \mathrm{mg} \cdot \mathrm{kg}^{-1} \cdot \mathrm{day}^{-1}\right)$ was chosen based on previous experience in spontaneously hypertensive rats, in which this dose controlled blood pressure and prevented target organ damage [40]. However, the optimally effective doses in diabetic nephropathy may differ from those in arterial hypertension. Therefore, comparative statements based on single dose groups are not valid, although there were no major systemic haemodynamic effects in the present study. Also, plasma ACE inhibition was almost complete in the ramipril as well as the AVE7688 group in a recent parallel study [19]. Nevertheless, further studies on the local renal level will be necessary to identify the specific mode of nephroprotection by AVE7688.

In addition to excretion, exogenous supply and endogenous formation of AGEs are important determinants of serum AGE levels. Chronic exposure of rats to high levels of exogenous AGE can induce morphological kidney damage $[33,37]$. In the present study, the composition of the chow was controlled and average daily food intake was similar across the groups, which excludes the confounding effects of exogenous AGEs. Also, the decrease in serum CML cannot be explained by a generally favourable influence of AVE7688 on glucose control, which could have ameliorated the metabolic stress. Therefore, we addressed potential compound-specific effects on the production of AGE in vitro. We found that the active metabolite of AVE7688 (AVE8048) but not that of ramipril (ramiprilat) inhibited the formation of CML and pentosidine in vitro. Recent investigations on antihypertensive drugs revealed that renin-angiotensin system inhibitors, such as ACE inhibitors and angiotensin II type 1 receptor blockers, also inhibited AGE formation in vitro and in vivo [41, 42]. Unlike established AGE inhibitors such as aminoguanidine, which trap reactive carbonyl precursors, the ACE inhibitors and angiotensin II type 1 receptor blockers, which do not have carbonyl-reactive functional groups, may inhibit the production of reactive carbonyl precursors by chelating transition metals and inhibiting various oxidative steps at both the pre- and the post-Amadori stage $[41,42]$. Therefore, we investigated the chelating potential of the active metabolites of AVE7688 (AVE8048) and ramipril (ramiprilat) and compared them with the AGE inhibitors pyridoxamine and tenilsetam [21], using an assay based on inhibition of copper-catalysed oxidation of ascorbic acid. Compared with ramiprilat, AVE8048 was a 500 -fold more potent inhibitor of copper-catalysed oxidation of ascorbate, which indicates its potential to inhibit the formation of AGEs directly through its chelating activity. Since both CML and pentosidine formation from glucose are affected by metal-catalysed glycoxidation reactions
$[43,44]$, the metal-chelating activity of AVE8048 may play an important role in the inhibition of AGE formation and provide additional protection to the diabetic kidney. Pharmacokinetic measurements have shown a certain degree of accumulation of the metabolites of both ramipril and AVE7688 in the kidney, resulting in concentrations that are 5- to 10-fold higher in the kidney than in plasma (data not shown), so that they may have a more pronounced local effect in the kidney. Cooper and colleagues [45] have demonstrated recently that the copper chelator trientine has a profound cardioprotective effect in diabetic rats. Although they did not measure renal function or AGEs, their data support the contribution of chelation in the superior renoprotective effect of AVE7688, whose active metabolite AVE8048 is also a potent copper chelator. At the same time, this drug also inhibited the formation of 3-DG-imidazolone, which is produced by a non-oxidative pathway, so that other mechanisms of action are probably also involved.

In summary, the present study suggests that AVE7688 provides renal protection beyond vasopeptidase inhibition by inhibiting the formation of 3-DG-imidazolone, pentosidine and CML. There is also evidence that increased renal CML clearance in association with improved renal function may contribute to reduced accumulation of the specific AGEs CML, pentosidine and 3-DG-imidazolone in diabetic nephropathy. Here we show for the first time that AVE7688 also acts as an AGE inhibitor in vitro, probably through its metal-chelating activity. We conclude that vasopeptidase inhibitors may have higher renoprotective potential than agents that only interfere with the reninangiotensin system, and that AGE inhibition may be one important contributor to this effect.

Acknowledgements This work was supported by Aventis Pharma Deutschland, a company of the Sanofi-Aventis group, as part of the Cardiovascular and Renal Endpoints in Diabetes (CARED) programme, and in part by USPHS grant DK-19971 to J. W. Barnes.

Duality of interest statement

Professors A. E. Busch and M. Bleich are previous employees of Aventis Pharma but currently have no financial interest in the company. C. Wihler, S. Schäfer and J. Huber are current employees of Aventis Pharma and have a financial interest in the company. The manuscript deals with pharmacological compounds that are either marketed (ramipril) or in development by Aventis Pharma. The authors confirm, however, that their association (as mentioned above) with Aventis Pharma has in no way affected the conduct and/or interpretation of experiments in this work.

J. W. Baynes is a member of the Scientific Advisory Board of BioStratum, a biotechnology company developing agents for the treatment of diabetic nephropathy.

\section{References}

1. Cooper ME (2001) Interaction of metabolic and haemodynamic factors in mediating experimental diabetic nephropathy. Diabetologia 44:1957-1972

2. Raj DS, Choudhury D, Welbourne TC, Levi M (2000) Advanced glycation end products: a nephrologist's perspective. Am J Kidney Dis 35:365-380

3. Brownlee M (1995) Advanced protein glycosylation in diabetes and aging. Annu Rev Med 46:223-234 
4. Miyata T, Izuhara Y, Sakai H, Kurokawa K (1999) Carbonyl stress: increased carbonyl modification of tissue and cellular proteins in uremia. Perit Dial Int 19(Suppl 2):S58-S61

5. Singh R, Barden A, Mori T, Beilin L (2001) Advanced glycation end-products: a review. Diabetologia 44:129-146

6. Heidland A, Sebekova K, Schinzel R (2001) Advanced glycation end products and the progressive course of renal disease. Am J Kidney Dis 38:S100-S106

7. Kislinger T, Fu C, Huber B et al (1999) N(epsilon)-(carboxymethyl)lysine adducts of proteins are ligands for receptor for advanced glycation end products that activate cell signaling pathways and modulate gene expression. J Biol Chem 274: 31740-31749

8. Bierhaus A, Schiekofer S, Schwaninger M et al (2001) Diabetes-associated sustained activation of the transcription factor nuclear factor-kappaB. Diabetes 50:2792-2808

9. Yeh CH, Sturgis L, Haidacher J et al (2001) Requirement for p38 and p44/p42 mitogen-activated protein kinases in RAGEmediated nuclear factor-kappaB transcriptional activation and cytokine secretion. Diabetes 50:1495-1504

10. Schleicher ED, Bierhaus A, Haring HU, Nawroth PP, Lehmann R (2001) Chemistry and pathobiology of advanced glycation end products. Contrib Nephrol (131):1-9

11. Schleicher ED, Wagner E, Nerlich AG (1997) Increased accumulation of the glycoxidation product $N$ (epsilon)-(carboxymethyl)lysine in human tissues in diabetes and aging. J Clin Invest 99:457

12. Tanji N, Markowitz GS, Fu C et al (2000) Expression of advanced glycation end products and their cellular receptor RAGE in diabetic nephropathy and nondiabetic renal disease. J Am Soc Nephrol 11:1656-1666

13. Kilhovd BK, Giardino I, Torjesen PA et al (2003) Increased serum levels of the specific AGE-compound methylglyoxalderived hydroimidazolone in patients with type 2 diabetes. Metabolism 52:163-167

14. Monnier VM, Sell DR, Nagaraj RH et al (1992) Maillard reaction-mediated molecular damage to extracellular matrix and other tissue proteins in diabetes, aging, and uremia. Diabetes 41 (Suppl 2):36-41

15. HOPE Study Investigators (2000) Effects of ramipril on cardiovascular and microvascular outcomes in people with diabetes mellitus: results of the HOPE study and MICRO-HOPE substudy. Lancet 355:253-259

16. Forbes JM, Cooper ME, Thallas V et al (2002) Reduction of the accumulation of advanced glycation end products by ACE inhibition in experimental diabetic nephropathy. Diabetes 51: 3274-3282

17. Davis BJ, Johnston CI, Burrell LM et al (2003) Renoprotective effects of vasopeptidase inhibition in an experimental model of diabetic nephropathy. Diabetologia 46:961-971

18. Wenzel UO, Wolf G, Jacob I et al (2003) Beneficial and adverse renal and vascular effects of the vasopeptidase inhibitor omapatrilat in renovascular hypertensive rats. Nephrol Dial Transplant 18:2005-2013

19. Schafer S, Linz W, Bube A et al (2003) Vasopeptidase inhibition prevents nephropathy in Zucker diabetic fatty rats. Cardiovasc Res 60:447-454

20. Drusch S, Faist V, Erbersdobler HF (1999) Determination of $N$ (epsilon)-(carboxymethyl)lysine in milk products by a modified reversed-phase HPLC method. Food Chem 65:547-553

21. Price DL, Rhett PM, Thorpe SR, Baynes JW (2001) Chelating activity of advanced glycation end-product inhibitors. J Biol Chem 276:48967-48972

22. Schafer S, Linz W, Vollert H et al (2004) The vasopeptidase inhibitor AVE7688 ameliorates Type 2 diabetic nephropathy. Diabetologia 47:98-103

23. Forbes JM, Cooper ME, Oldfield MD, Thomas MC (2003) Role of advanced glycation end products in diabetic nephropathy. J Am Soc Nephrol 14:S254-S258
24. Niwa T, Katsuzaki T, Miyazaki S et al (1997) Immunohistochemical detection of imidazolone, a novel advanced glycation end product, in kidneys and aortas of diabetic patients. J Clin Invest 99:1272-1280

25. Suzuki D, Miyata T, Saotome N et al (1999) Immunohistochemical evidence for an increased oxidative stress and carbonyl modification of proteins in diabetic glomerular lesions. J Am Soc Nephrol 10:822-832

26. Wagner Z, Wittmann I, Mazak I et al (2001) N(epsilon)-(carboxymethyl)lysine levels in patients with type 2 diabetes: role of renal function. Am J Kidney Dis 38:785-791

27. Koschinsky T, He CJ, Mitsuhashi T et al (1997) Orally absorbed reactive glycation products (glycotoxins): an environmental risk factor in diabetic nephropathy. Proc Natl Acad Sci U S A 94:6474-6479

28. Morcos M, Sayed AA, Bierhaus A et al (2002) Activation of tubular epithelial cells in diabetic nephropathy. Diabetes 51: 3532-3544

29. Thornalley PJ, Battah S, Ahmed N et al (2003) Quantitative screening of advanced glycation endproducts in cellular and extracellular proteins by tandem mass spectrometry. Biochem J 375:581-592

30. Wilkinson-Berka JL, Kelly DJ, Koerner SM et al (2002) ALT946 and aminoguanidine, inhibitors of advanced glycation, improve severe nephropathy in the diabetic transgenic (mREN2)27 rat. Diabetes 51:3283-3289

31. Agardh CD, Stenram U, Torffvit O, Agardh E (2002) Effects of inhibition of glycation and oxidative stress on the development of diabetic nephropathy in rats. J Diabetes Its Complicat 16: 395-400

32. Forbes JM, Soulis T, Thallas V et al (2001) Renoprotective effects of a novel inhibitor of advanced glycation. Diabetologia $44: 108-114$

33. He C, Sabol J, Mitsuhashi T, Vlassara H (1999) Dietary glycotoxins: inhibition of reactive products by aminoguanidine facilitates renal clearance and reduces tissue sequestration. Diabetes 48:1308-1315

34. Shimoike T, Inoguchi T, Umeda F, Nawata H, Kawano K, Ochi H (2000) The meaning of serum levels of advanced glycosylation end products in diabetic nephropathy. Metabolism 49:1030 1035

35. Makita Z, Bucala R, Rayfield EJ et al (1994) Reactive glycosylation endproducts in diabetic uraemia and treatment of renal failure. Lancet 343:1519-1522

36. Gugliucci A, Bendayan M (1996) Renal fate of circulating advanced glycated end products (AGE): evidence for reabsorption and catabolism of AGE-peptides by renal proximal tubular cells. Diabetologia 39:149-160

37. Sebekova K, Faist V, Hofmann T, Schinzel R, Heidland A (2003) Effects of a diet rich in advanced glycation end products in the rat remnant kidney model. Am J Kidney Dis 41:S48-S51

38. Soulis T, Cooper ME, Vranes D, Bucala R, Jerums G (1996) Effects of aminoguanidine in preventing experimental diabetic nephropathy are related to the duration of treatment. Kidney Int 50:627-634

39. Thallas-Bonke V, Lindschau C, Rizkalla B et al (2004) Attenuation of extracellular matrix accumulation in diabetic nephropathy by the advanced glycation end product cross-link breaker ALT-711 via a protein kinase C-alpha-dependent pathway. Diabetes 53:2921-2930

40. Linz W, Jessen T, Becker RHA, Scholkens BA, Wiemer G (1997) Long-term ACE inhibition doubles lifespan of hypertensive rats. Circulation 96:3164-3172

41. Miyata T, van Ypersele de Strihou C, Ueda Y et al (2002) Angiotensin II receptor antagonists and angiotensin-converting enzyme inhibitors lower in vitro the formation of advanced glycation end products: biochemical mechanisms. J Am Soc Nephrol 13:2478-2487 
42. Nangaku M, Miyata T, Sada T et al (2003) Anti-hypertensive agents inhibit in vivo the formation of advanced glycation end products and improve renal damage in a type 2 diabetic nephropathy rat model. J Am Soc Nephrol 14:1212-1222

43. Saxena AK, Saxena P, Wu X, Obrenovich M, Weiss MF, Monnier VM (1999) Protein aging by carboxymethylation of lysines generates sites for divalent metal and redox active copper binding: relevance to diseases of glycoxidative stress. Biochem Biophys Res Commun 260:332-338
44. Weiss MF, Erhard P, Kader-Attia FA et al (2000) Mechanisms for the formation of glycoxidation products in end-stage renal disease. Kidney Int 57:2571-2585

45. Cooper GJS, Phillips ARJ, Choong SY et al (2004) Regeneration of the heart in diabetes by selective copper chelation. Diabetes 53:2501-2508 